\title{
Fatal outcome of malignant phyllodes tumor of the breast in pregnancy: a case and literature review
}

\author{
Wen-Xiang Zhang ${ }^{1 \#}$, Xiang-Yi Kong ${ }^{1 \#}$, Jie Zhai ${ }^{1 \#}$, Yi Fang ${ }^{1}$, Yan Song ${ }^{2}$, Jing Wang ${ }^{1}$ \\ ${ }^{1}$ Department of Breast Surgical Oncology, National Cancer Center/National Clinical Research Center for Cancer/Cancer Hospital, Chinese \\ Academy of Medical Sciences and Peking Union Medical College, Beijing, China; ${ }^{2}$ Department of Pathology, National Cancer Center/National \\ Clinical Research Center for Cancer/Cancer Hospital, Chinese Academy of Medical Sciences and Peking Union Medical College, Beijing, China \\ \#These authors contribute equally to this work. \\ Correspondence to: Jing Wang; Yan Song; Yi Fang. Department of Breast Surgical Oncology, Department of Pathology, National Cancer Center/ \\ National Clinical Research Center for Cancer/Cancer Hospital, Chinese Academy of Medical Sciences and Peking Union Medical College, Beijing \\ 100021, China. Email: wwwjjj1234@vip.sina.com; songyanerer@hotmail.com; fangyi0501@vip.sina.com.
}

\begin{abstract}
Phyllodes tumor of the breast (PTB) is a rare fibroepithelial breast neoplasm that accounts for less than $1 \%$ of breast tumors. Only a few cases related to pregnancy have been reported. It is not known how pregnancy affects the diagnosis, treatment and prognosis of breast tumors. Here we report a case of a 38-year-old female patient with a small, mobile palpable lump in the left breast for about 15 years. it was considered a benign lesion and no surgical treatment was performed at the beginning. The left breast mass became larger suddenly during pregnancy, However, she did not see the doctor and receive any treatment in time. The lump was resected one year after labor and confirmed to be malignant phyllodes of the breast by histopathology and immunohistochemistry. Unfortunately, local recurrence occurred within six months after the first operation, and lung metastasis occurred eight months later. And this patient finally died 13 months after the operation due to tumor progression. This is the first report of pregnancy-related malignant PTB, with local recurrence and distant metastasis in a short period. This case report highlights a situation: the patient should be diagnosed early and treated in time when she has a previous breast fibroadenoma, but suddenly increased during pregnancy.
\end{abstract}

Keywords: Malignant phyllodes tumor (MPT); recurrence; pregnancy; case report and literature review

Submitted May 31, 2020. Accepted for publication Nov 28, 2020.

doi: 10.21037 /gs-20-538

View this article at: http://dx.doi.org/10.21037/gs-20-538

\section{Introduction}

Phyllodes tumor of the breast (PTB) is a rare type of biphasic fibroepithelial neoplasms that include the breast stroma and epithelium, which comprise only 0.3 to 1 percent of all breast tumors in women (1). The World Health Organization (WHO) classified phyllodes tumors into benign, borderline, and malignant categories based on histological characteristics. It mainly occurs in female patients and can be present at any age from puberty to menopause. Phyllodes tumor may present during pregnancy like all other breast tumors, but this is underrepresented in the literature $(2,3)$.
In this report, we report a female patient with malignant phyllodes tumor (MPT) of the left breast who underwent resection of right breast fibroadenoma 15 years ago. The postoperative pathology supported this result. The left breast mass considered benign lesions (BI-RADS3). No surgical treatment was performed. The left breast mass became larger suddenly during pregnancy in 2015 . However, she did not undergo any treatment and went to see a doctor after pregnancy and breastfeeding. Local recurrence occurred within six months after the first operation, and lung metastasis occurred eight months later. Short-term recurrence and metastasis are not frequent. Which is different from the cases reported previously. 

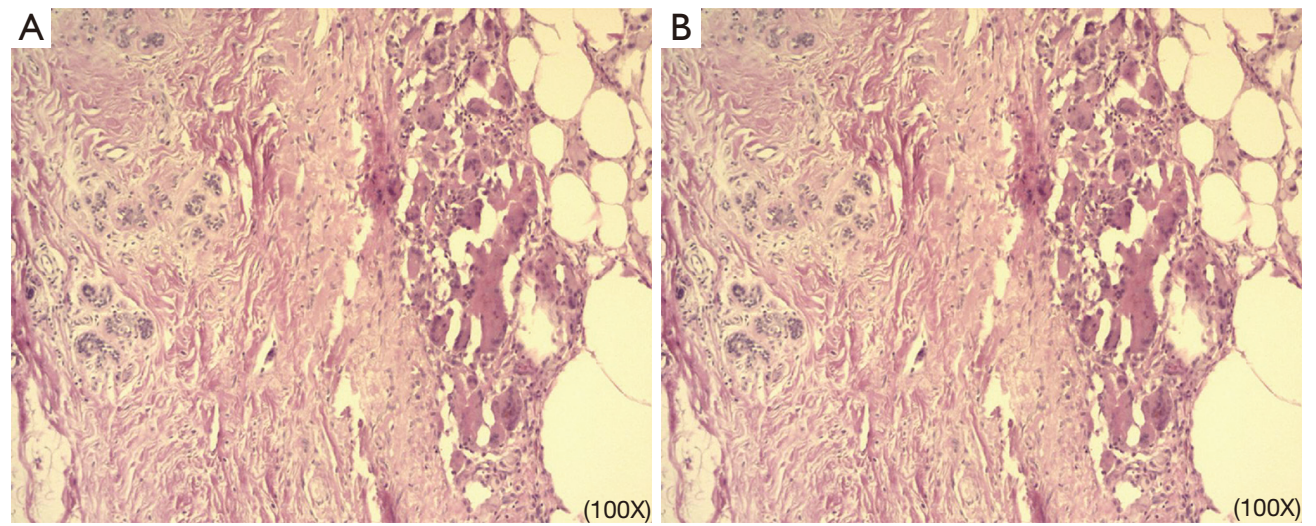

Figure $1 \mathrm{H} \& \mathrm{E}$ staining of the tumor. (A) The surgical area showed fibrous tissue hyperplasia, a multicore giant cell response accompanied by inflammatory cell infiltration (H\&E staining; magnification, $\times 100$ ). (B) The surgical area demonstrated the formation of fibroadenomas (H\&E staining; magnification, $\times 100)$. H\&E staining, hematoxylin-eosin staining.

Secondly, the treatment process (secondary surgery, local radiotherapy, adjustment of a variety of chemotherapy and targeted therapy, endocrine therapy) is described in detail, and the complexity of the treatment process is also rare. We report such a particular case in order to explore the effect of pregnancy and breastfeeding on the occurrence and development of MPTs of the breast. Secondly, especially for the initially suspected breast fibroadenoma, but suddenly increased in a short time. We must warn about the possibility of phyllodes breast tumors. We present the following article/case in accordance with the CARE reporting checklist (available at http://dx.doi.org/10.21037/ gs-20-538).

\section{Case presentation}

A 38-year-old woman was admitted to the hospital with a complaint of a growing left breast mass for three years. She reported that 15 years ago, she had nodules on both sides of her breast and that the right breast mass was relatively large and surgically removed. Furthermore, histopathological examination revealed benign fibroadenomas. Because the left breast nodule was too small, it was considered a benign lesion by color Doppler ultrasound, and no surgical treatment was performed. After that, a regular reexamination of the breast by ultrasonography was conducted. However, the left breast mass became larger suddenly in $2015\left(3 \times 3 \times 2 \mathrm{~cm}^{3}\right)$, but she did not see a doctor due to pregnancy (at eight weeks gestation). Then the left breast tumor $\left(6 \times 5 \times 4 \mathrm{~cm}^{3}\right)$ resection was not performed until March 8, 2018. A malignant breast phyllodes tumor was then demonstrated with histopathological examination. It is uncertain whether there is tumor residue at the margins, and a total remaining breast resection was performed with the patient's permission on March 28, 2018. No tumor residue in the left breast tissue with the histopathological examination (Figure 1).

Unfortunately, in July 2018, the patient found a mass under the left armpit. One month later, enlarged axillary mass resection and axillary lymph node biopsy were performed. Postoperative pathology showed a recurrence of malignant breast tumors, and no tumor metastasis was found in the axillary lymph node (Figure 2). Then left chest wall and armpit radiotherapy was performed since September 2018. Half a month after the last radiotherapy (October 30, 2018), left axillary multiple stable nodes appeared, and a recurrence was considered.

On December 3, 2018, the chest CT image supported a lung metastasis diagnosis, evidenced by multiple small nodules in both lungs. Among them, the largest one was $0.6 \mathrm{~cm}$. Then a series of chemotherapy, targeted therapy, endocrine therapy was conducted (Table 1). Unfortunately, in the treatment process, the tumor has been progressing, resulting in multiple organ failure due to hypoproteinemia, pernicious anemia, electrolyte disorder, infection, etc. and finally died of invalid treatment on July 16, 2019.

All procedures performed in studies involving human participants were in accordance with the ethical standards of the institutional and/or national research committee(s) and with the Helsinki Declaration (as revised in 2013). Written informed consent was obtained from the guardian of patients for publication of this case report and any 

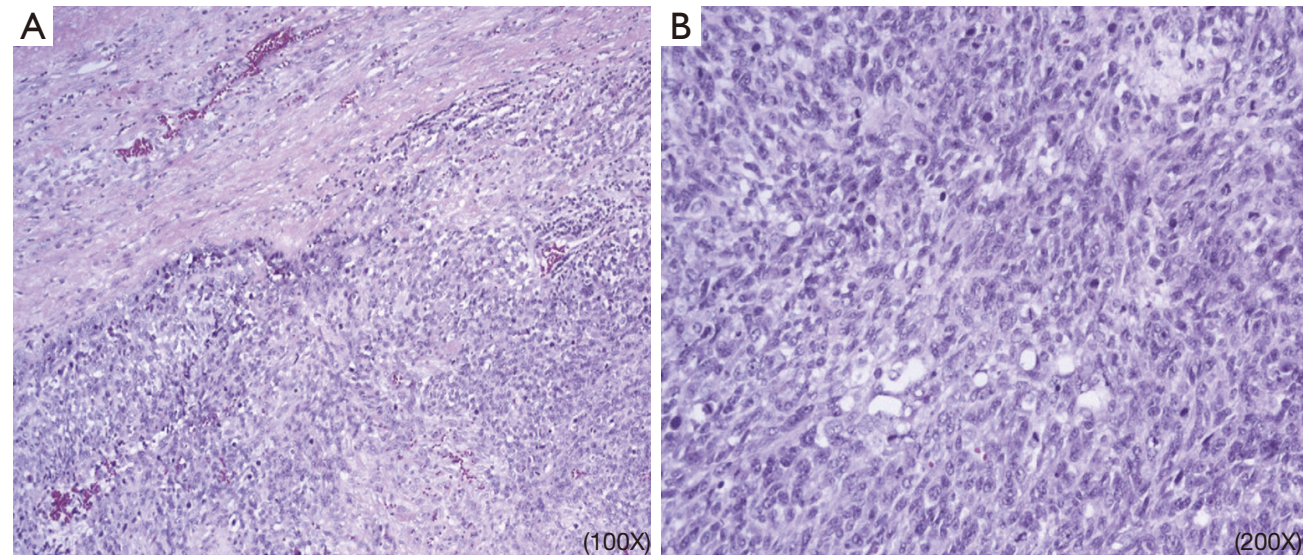

Figure $2 \mathrm{H} \& \mathrm{E}$ staining of the tumor. Malignant phyllodes tumor, consistent with recurrence of breast malignant phyllodes tumor, visible necrosis, mitotic phase easy to see (>5/HPF). (A) (H\&E staining; magnification, $\times 100)$; (B) (H\&E staining; magnification, $\times 200)$. H\&E staining, hematoxylin-eosin staining; HPF, high power field.

Table 1 Disease development process and the treatment line

\begin{tabular}{lll}
\hline Time & Disease development & Treatment \\
\hline 2018.12 .21 & Local recurrence, acute double lung metastasis & Chemotherapy (two cycles) $^{\dagger}$ \\
2019.02 .15 & Axillary swelling and collapse & Targeted therapy $^{\ddagger}$ \\
2019.03 .30 & Tumor progression & Chemotherapy (three cycles) and targeted therapy $^{\S}$ \\
2019.06 .26 & Tumor progression & Change chemotherapy plan" \\
2109.07 .09 & Chemotherapy intolerable & Endocrine therapy \\
2019.07 .16 & Anemia, infection, heart failure, invalid treatment and death & \\
\hline
\end{tabular}

${ }^{\dagger}$, albumin paclitaxel + cisplatin; ${ }^{\ddagger}$, lapatinib; ${ }^{\S}$, apatinib + epirubicin + ifosfamide; ${ }^{\text {? }}$, gemcitabine + bevacizumab; ', etoposide.

accompanying images.

\section{Discussion}

MPT is a relatively rare tumor, constituting $25 \%$ of all PTB (4). Only eight previous cases of MPT in pregnancy were found, information from each case report was summarized in Table 2. The median tumor size was $10.3 \mathrm{~cm}$, ranging from 1.7 to 21 $\mathrm{cm}$, it characteristically has grown very rapidly and attained large sizes by the time of presentation. Majority of patients was in the second half of pregnancy, six of these patients presented with unilateral phyllodes tumors $(2,3,5-8)$, two patient had bilateral disease $(9,10)$. All patients were actively treated after discovery. However, the follow-up failed to perform, and adjuvant therapy was not described in detail.

Our case is unique in that it is the first report of a patient with a large lump tumor presenting early in the first trimester of pregnancy, but it was not actively treated when the tumor increased during pregnancy because of the patient's insufficient knowledge of the disease, the diagnosis and treatment started only after two years. Unfortunately, local recurrence and metastasis of lungs occurred in a short period, which eventually led to death. In the literature, only one other patient presented during the first trimester with a $4 \mathrm{~cm}$ tumor treated with segmental mastectomy at 11 weeks gestation (2). At eight months of follow-up, local or distant recurrence did not occur. The treatment of these two patients at different times led to different prognosis. Such an outcome has caused great curiosity as to what caused the disease to develop so rapidly, is it that the phyllodes tumor itself is developing rapidly, or the hormone changes in the body caused by pregnancy and lactation cause the disease to deteriorate.

Hormonal activity during pregnancy induces proliferative 
Table 2 Case reports of malignant phyllodes tumor occurring during pregnancy

\begin{tabular}{|c|c|c|c|c|c|c|c|c|c|c|}
\hline $\begin{array}{l}\text { First } \\
\text { author }\end{array}$ & $\begin{array}{c}\text { Published } \\
\text { year }\end{array}$ & $\begin{array}{l}\text { Age, } \\
\text { year }\end{array}$ & $\begin{array}{l}\text { Gestation } \\
\text { presented }^{\dagger}\end{array}$ & Size $(\mathrm{cm})$ & $\begin{array}{l}\text { Rapid } \\
\text { growth }\end{array}$ & $\begin{array}{c}\text { History of } \\
\text { fibroadenoma }\end{array}$ & $\begin{array}{l}\text { Operation } \\
\text { period }\end{array}$ & $\begin{array}{l}\text { Surgical } \\
\text { approach }\end{array}$ & Pathology & Follow up \\
\hline Blaker (2) & 2010 & 27 & $9^{\text {th }}$ week & 4 & No & Yes & $\begin{array}{l}11 \text { weeks } \\
\text { gestation }\end{array}$ & WLE & Malignant & $\begin{array}{l}\text { At } 8 \text { months of } \\
\text { follow-up, local or } \\
\text { distant recurrence } \\
\text { did not occur }\end{array}$ \\
\hline Pacchiarotti (3) & 2011 & 41 & $17^{\text {th }}$ week & 6 & Yes & Yes & $\begin{array}{l}\text { During } \\
\text { pregnancy }\end{array}$ & WLE & Malignant & $\begin{array}{l}\text { The patient } \\
\text { delivered by } \\
\text { c-section at } \\
36 \text { weeks }\end{array}$ \\
\hline Simpson (5) & 2007 & 30 & $20^{\text {th }}$ week & 17 & Yes & Yes & $\begin{array}{l}22 \text { weeks } \\
\text { gestation }\end{array}$ & WLE & Malignant & $\begin{array}{l}\text { The patient } \\
\text { delivered by } \\
\text { c-section at } \\
32 \text { weeks }\end{array}$ \\
\hline Ray (7) & 2011 & 24 & $36^{\text {th }}$ week & NR & Yes & Small lump & $\begin{array}{l}8 \text { weeks after } \\
\text { labor }\end{array}$ & SM & Malignant & NR \\
\hline Kelten (8) & 2016 & 37 & $\begin{array}{l}4 \text { weeks } \\
\text { after labor }\end{array}$ & 13 & Yes & NR & $\begin{array}{l}4 \text { weeks after } \\
\text { labor }\end{array}$ & SM & Malignant & NR \\
\hline Mrad (9) & 2000 & 32 & NR & $\begin{array}{l}9 \text { (right), } \\
21 \text { (left) }\end{array}$ & NR & NR & NR & $\begin{array}{l}\text { WLE } \\
\text { (right); } \\
\text { SM (left) }\end{array}$ & $\begin{array}{l}\text { Benign (right); } \\
\text { malignant (left) }\end{array}$ & $\begin{array}{l}\text { The patient was } \\
\text { free of disease after } \\
17 \text { months of } \\
\text { surgery }\end{array}$ \\
\hline
\end{tabular}

${ }^{\dagger}$, seeing a doctor for the first time; ${ }^{\ddagger}$, two operations on the left breast, firstly, wire-guided lumpectomy of the left breast were carried out, the second surgery were carried out with subcutaneous adenectomy and immediate breast reconstruction. NR, not reported; SA+IBR, subcutaneous adenectomy and immediate breast reconstruction; SM, simple mastectomy; WLE, wide local excision.

changes of breast glandular tissue consisting of lobular and alveolar growth, and this may be the cause of rapid growth in MPT during pregnancy. Rapid enlargement of MPT in pregnancy has been previously reported. As seen in our case, the left breast mass suddenly increased in 2015 , and the mass continued to increase during pregnancy and lactation. A previous study has found that tumor size is a prognostic factor that affects the recurrence of the disease (11). The maximum diameter of the tumor is $\geq 10 \mathrm{~cm}$ compared with $<10 \mathrm{~cm}$; the former is four times more likely to recur than the latter (12). The prognosis was also affected by the speculative increased risk of metastasis resulting from the increased blood supply of breast tissue during pregnancy. However, due to the relatively small number of cases with MPT in pregnancy, more research is needed to confirm whether changes in hormone levels caused by pregnancy are related to the occurrence and development of PTB.

An interesting fact about this case is our patient's prior history of one excised fibroadenomas in the right breast, and the left breast mass was too small to be treated surgically. Consistently, it was more likely to be a 
fibroadenoma through imaging examination. However, a malignant breast phyllodes tumor was then founded. This may indicate that the formation of PTB may be related to fibroadenomas. Of the eight reported cases of MPT during pregnancy in the literature, 4 reported a history of biopsy of a previous breast mass, all of these species for fibroadenoma $(2,3,5,10)$, which is unique to this small series of pregnant patients. Current research has always considered that epithelial-mesenchymal interactions play an essential role in the progression of phyllodes tumor. Kuijper et al. found that fibroadenoma can progress in an epithelial direction to carcinoma in situ (CIS) and a stromal direction to phyllodes tumor (13). Other previous studies have found that fibroadenoma and phyllodes tumor show continuity in gross and microscopic morphology, and many patients have the above two lessons at the same time or at different times, and some tumors have the histological characteristics of fibroadenoma and phyllodes tumor at the same time (14). These findings support the hypothesis that PTB may originate from fibroadenomas. Furthermore, the examination of the cases of phyllodes tumors presenting in pregnancy suggests that pregnancy could exacerbate that risk even further.

Besides, we have recorded the entire treatment process in detail, including secondary surgery, local radiotherapy, various chemotherapy, and targeted therapy adjustments, and endocrine therapy. However, these treatments have not delayed the progression of the disease, which may be related to the delayed diagnosis and treatment of the disease and its tumor biological behavior. PTB are biphasic tumors, and their biological behavior is difficult to predict. The uncertainty of preoperative diagnosis provides a particular obstacle for the formulation of a reasonable surgical treatment plan. The negative margin is an independent prognostic factor of disease-free survival (DFS) and local recurrence rate of PTB. To make sure the cutting edge is negative, expanding the resection area is a fundamental principle regardless of its classification. Generally, the distance between the cutting edge and the tumor is more than $1 \mathrm{~cm}$. A simple mastectomy is preferred if negative margins cannot be achieved. For how to deal with axillary lymph nodes, many authors consider that axillary lymph node dissection (ALND) is not required for malignant PT due to the extremely low incidence of nodal involvement $(15,16)$. In a multicenter study including 1,035 patients, ALND was carried out in 9\% of patients and axillary lymph node involvement was demonstrated in nine patients (17). Because the possibility of axillary nodal involvement is not always negligible, SLN biopsy may be useful to distinguish localized malignant PT without regional disease from malignant $\mathrm{PT}$ with regional disease. To note that, like our case, this patient had tumor excision followed by total mastectomy. Neither sentinel lymph node biopsy nor ALND was performed. Several months later, she developed axillary recurrence followed by distant metastases. The local recurrence rates of PT published by the WHO in 2012 were: benign $10 \%$ to $17 \%$, borderline $14 \%$ to $25 \%$, and malignant $23 \%$ to $30 \%$ (18). The 5 -year DFS of PTB were: benign $95.7 \%$, borderline $82.5 \%$, malignant $72.6 \%$, and distant metastasis rate of malignant PT was 25-40\% (19). Therefore, in clinical work, we must recognize that both benign and MPTs can recur locally and have the potential for metastasis. Local recurrence may be related to the pathological classification of PT, the surgical method, and the degree of the resection margin. As the number of local recurrences increases, the histological grade of the tumor may increase.

Because of the low incidence rate of phyllodes tumors, there is no prospective study to illustrate the effectiveness of radiotherapy. Previous studies using the Surveillance, Epidemiology, and End Results Program (SEER) data [1983-2013] showed that adjuvant radiotherapy did not affect survival, regardless of the mode of surgery (15). Gnerlich et al. (20) found that adjuvant radiotherapy can reduce the local recurrence rate and however, it has no significant effect on patients' overall survival (OS) and DFS. Also, adjuvant chemotherapy is controversial since there is little clinical benefit. neoadjuvant doxorubicin plus dacarbazine versus has been reported in a small observational biased trial with no benefit of relapse-free survival. Routine chemotherapy is not encouraged in the standard treatment for MPT. It can be considered in extreme circumstances such as large tumors, or involvement of secondary structures like the chest wall. However, the benefit of such a situation is not well documented or recommended by the national comprehensive cancer network (NCCN) guidelines.

\section{Conclusions}

Phyllodes tumors should be accurately recognized and effectively treated in time, especially during pregnancy, as this tumor is prone to recur and distant metastasis. Furthermore, patients with breast fibroadenomas suddenly increased during pregnancy should be valued in order to make an early diagnosis and distinction of phyllodes 
tumors. In terms of treatment, surgical treatment is the first recommended, and the effect of radiotherapy and chemotherapy is little. To reduce the risk of tumor recurrence, a standard therapeutic strategy for phyllodes tumor is urgent. More analytical clinical studies are worthwhile in the future.

\section{Acknowledgments}

Funding: This study was supported by the National Natural Science Foundation of China (No. 81872160), Capital Public Health Education, Beijing Science, and Technology Program of China (No. Z171100000417028), and Peking Union Medical College Youth Research Funds of China (No. 3332015157).

\section{Footnote}

Reporting Checklist: The authors have completed the CARE reporting checklist. Available at http://dx.doi.org/10.21037/ gs-20-538

Conflicts of Interest: All authors have completed the ICMJE uniform disclosure form (available at http://dx.doi. org/10.21037/gs-20-538). The authors have no conflicts of interest to declare.

Ethical Statement: The authors are accountable for all aspects of the work in ensuring that questions related to the accuracy or integrity of any part of the work are appropriately investigated and resolved. The information has been approved by ethical review by the ethics committee of Cancer Hospital Chinese Academy of Medical Sciences. All procedures performed in studies involving human participants were in accordance with the ethical standards of the institutional and/or national research committee(s) and with the Helsinki Declaration (as revised in 2013). Written informed consent was obtained from the guardian of patients for publication of this case report and any accompanying images. A copy of the written consent is available for review by the Editor-in-Chief of this journal.

Open Access Statement: This is an Open Access article distributed in accordance with the Creative Commons Attribution-NonCommercial-NoDerivs 4.0 International License (CC BY-NC-ND 4.0), which permits the noncommercial replication and distribution of the article with the strict proviso that no changes or edits are made and the original work is properly cited (including links to both the formal publication through the relevant DOI and the license). See: https://creativecommons.org/licenses/by-nc-nd/4.0/.

\section{References}

1. Assi H, Salem R, Sukhon F, et al. Phyllodes tumors of the breast treated in a tertiary health care center: case series and literature review. J Int Med Res 2020;48:300060518803530.

2. Blaker KM, Sahoo S, Schweichler MR, et al. Malignant phylloides tumor in pregnancy. Am Surg 2010;76:302-5.

3. Pacchiarotti A, Frati P, Caserta D, et al. First case of transformation for breast fibroadenoma to high-grade malignant cystosarcoma in an in vitro fertilization patient. Fertil Steril 2011;96:1126-7.

4. Zhang Y, Kleer CG. Phyllodes Tumor of the Breast: Histopathologic Features, Differential Diagnosis, and Molecular/Genetic Updates. Arch Pathol Lab Med 2016;140:665-71.

5. Simpson SA, Redstone J, Aziz MS, et al. Large malignant phyllodes tumor with rapid growth during pregnancy: images of a case. Breast J 2007;13:620-1.

6. Nejc D, Pasz-Walczak G, Piekarski J, et al. Astonishingly rapid growth of malignant cystosarcoma phyllodes tumor in a pregnant woman--a case report. Int J Gynecol Cancer 2008;18:856-9.

7. Ray S, Basak S, Das S, et al. Malignant phylloides tumor of breast in a pregnant woman with coincidental nulliparous vaginal prolapse. Iran J Med Sci 2011;36:315-7.

8. Gentile LF, Gaillard WF, Wallace JA, et al. A Case of a Giant Borderline Phyllodes Tumor Early in Pregnancy Treated with Mastectomy and Immediate Breast Reconstruction. Breast J 2016;22:683-7.

9. Mrad K, Driss M, Maalej M, et al. Bilateral cystosarcoma phyllodes of the breast: a case report of malignant form with contralateral benign form. Ann Diagn Pathol 2000;4:370-2.

10. Hernanz F, Gonzalez-Noriega M, Arozamena B, et al. Bilateral synchronous breast malignant phyllodes in a pregnant woman. Breast J 2018;24:412-3.

11. Co M, Chen C, Tsang JY, et al. Mammary phyllodes tumour: a 15-year multicentre clinical review. J Clin Pathol 2018;71:493-7.

12. Zeng S, Zhang X, Yang D, et al. Effects of adjuvant radiotherapy on borderline and malignant phyllodes tumors: A systematic review and meta-analysis. Mol Clin 
Oncol 2015;3:663-71.

13. Kuijper A, Buerger H, Simon R, et al. Analysis of the progression of fibroepithelial tumours of the breast by PCR-based clonality assay. J Pathol 2002;197:575-81.

14. Mangi AA, Smith BL, Gadd MA, et al. Surgical management of phyllodes tumors. Arch Surg 1999;134:487-92; discussion 492-3.

15. Kim YJ, Kim K. Radiation therapy for malignant phyllodes tumor of the breast: An analysis of SEER data. Breast 2017;32:26-32.

16. Guillot E, Couturaud B, Reyal F, et al. Management of phyllodes breast tumors. Breast J 2011;17:129-37.

17. Gullett NP, Rizzo M, Johnstone PA. National surgical

Cite this article as: Zhang WX, Kong XY, Zhai J, Fang Y, Song Y, Wang J. Fatal outcome of malignant phyllodes tumor of the breast in pregnancy: a case and literature review. Gland Surg 2021;10(1):371-377. doi: 10.21037/gs-20-538 patterns of care for primary surgery and axillary staging of phyllodes tumors. Breast J 2009;15:41-4.

18. Mitus JW, Blecharz P, Jakubowicz J, et al. Phyllodes tumors of the breast. The treatment results for 340 patients from a single cancer centre. Breast 2019;43:85-90.

19. Narayanakar RP, Gangaiah DM, Althaf S, et al. Cystosarcoma phyllodes: Pathological enigma: A retrospective review of 162 cases. Indian J Cancer 2015;52:365-8.

20. Gnerlich JL, Williams RT, Yao K, et al. Utilization of radiotherapy for malignant phyllodes tumors: analysis of the National Cancer Data Base, 1998-2009. Ann Surg Oncol 2014;21:1222-30. 